\title{
Spontaneous formation of a macroscopically extended coherent state
}

\author{
C. Braggio $\odot,{ }^{1, *}$ F. Chiossi, ${ }^{1}$ G. Carugno, ${ }^{1}$ A. Ortolan $\odot,{ }^{2}$ and G. Ruoso $\odot^{2}$ \\ ${ }^{1}$ Dipartimento di Fisica e Astronomia, Università di Padova and INFN, I-35131 Padova, Italy \\ ${ }^{2}$ INFN, Laboratori Nazionali di Legnaro, I-35020 Legnaro, Italy
}

(Received 11 March 2020; accepted 15 June 2020; published 13 July 2020)

\begin{abstract}
It is a straightforward result of electromagnetism that dipole oscillators radiate more strongly when they are synchronized and that if there are $N$ dipoles, then the overall emitted intensity scales with $N^{2}$. In atomic physics, such an enhanced radiative property appears when coherence among two-level identical atoms is established and is well known as "superradiance." In superfluorescence, atomic coherence develops via a self-organization process stemming from the common radiated field, starting from an incoherently prepared population inversion. In this work we establish the experimental conditions for formation of a macroscopic dipole via superfluorescence, involving the remarkable number of $4 \times 10^{12}$ atoms. Self-driven atom dynamics, without the mediation of cavity QED nor quantum dots or quantum well structures, is observed in a cryogenically cooled rare-earth doped material. We present clear evidence of a decay rate that is enhanced by more than one million times compared to that of independently emitting atoms.
\end{abstract}

DOI: 10.1103/PhysRevResearch.2.033059

\section{INTRODUCTION}

The paradigm effect for collective behavior in quantum optics is superradiance (SR), extensively studied both theoretically and experimentally starting from its prediction by Dicke in 1954 [1]. A strong radiative coupling is established among $N$ excited atoms that are indistinguishable owing to their being confined within a small volume $V<\lambda^{3}$, where $\lambda$ is the radiated field wavelength. The correlation among atoms gives rise to a macroscopic dipole, whereby each individual dipole accelerates the transition rate of the other emitters according to the "Dicke ladder" representation. Signatures of this effect are then to be sought in the natural $N$-squared scaling of the emission intensity by an array of phase-locked identical dipoles $N$ or in the temporal dynamics of their emission, described by sech-squared shape, intense coherent photon pulses.

An extension of the SR concept to much larger volumes $\left(V \gg \lambda^{3}\right)$ has been later theoretically analyzed [2], thus implying the possibility of experimentally accomplishing a proportionally larger number of atoms $N$.

A cooperative spontaneous emission (i.e., radiation rate proportional to $N^{2}$ ) can also be obtained from an atomic system initially excited with zero macroscopic dipole moment, as is the case in superfluorescence (SF) [3]. First demonstrated in a gas [4] and later in condensed matter systems [5], its potential is currently being investigated in the fields of ultranarrow linewidth laser development for fundamental tests in

\footnotetext{
*caterina.braggio@unipd.it

Published by the American Physical Society under the terms of the Creative Commons Attribution 4.0 International license. Further distribution of this work must maintain attribution to the author(s) and the published article's title, journal citation, and DOI.
}

physics [6-10] and for the development of devices enabling entangled multi-photon quantum light sources $[11,12]$. Such an enhanced radiative property is hard to observe in condensed matter compared to atomic and molecular gases (see Ref. [13] and references therein) due to stringent requirements that the atoms be indistinguishable and decoupled from their environment and that their density is high. In terms of spectroscopic properties of the material these demands translate to the following: small inhomogeneous line-broadening, small atomic dephasing rate, and large-enough inversion density. In the optical regime strong light-matter coupling via SF has been recently accomplished in nanostructured materials $[11,13-$ 16], involving small atom number and short collective state lifetime (approximately picoseconds).

Our work pushes into a new regime, demonstrating spontaneous formation of a macroscopic dipole composed of the remarkable number of atoms $N \simeq 4 \times 10^{12}$ in erbium-doped yttrium orthosilicate (Er:YSO, $\mathrm{Er}^{3+}: \mathrm{Y}_{2} \mathrm{SiO}_{5}$ ). This optical material exhibits the narrowest homogeneous linewidths and the longest coherence lifetimes [17] and is widely investigated for spectral hole burning applications $[18,19]$, cavity QED [20,21], and the reversible, coherent conversion of microwave photons into the optical telecom C band around $1.54 \mu \mathrm{m}$ [22]. Prior to our work, superfluorescence from bulk crystals had been demonstrated for $\mathrm{O}_{2}^{-}$centers in an alkali halide crystal $[5,23]$, even though the reported pulsed emission is clearly a superposition of pulses generated by small subsystems of correlated molecules [24]. The lifetime of the macroscopic quantum state we demonstrate is $\sim 100 \mathrm{~ns}$, exceeding previously reported values in solid state. This property and its high atom number might determine a nonvanishing probability of photon superabsorption $[25,26]$ if the level that superfluoresces is probed by an intense laser field. Beyond being interesting by its very nature, a superabsorber is sensitive to low microwave and light levels, a desirable feature in the context of quantum sensors development for future scientific instruments. The 
(a)

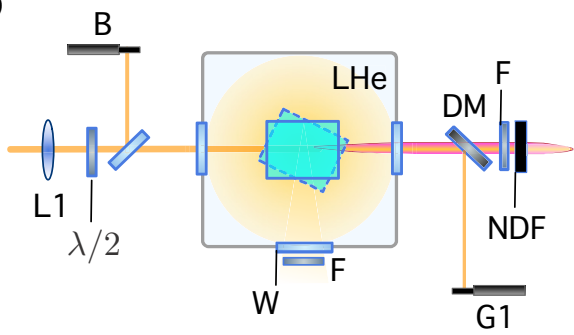

(b)
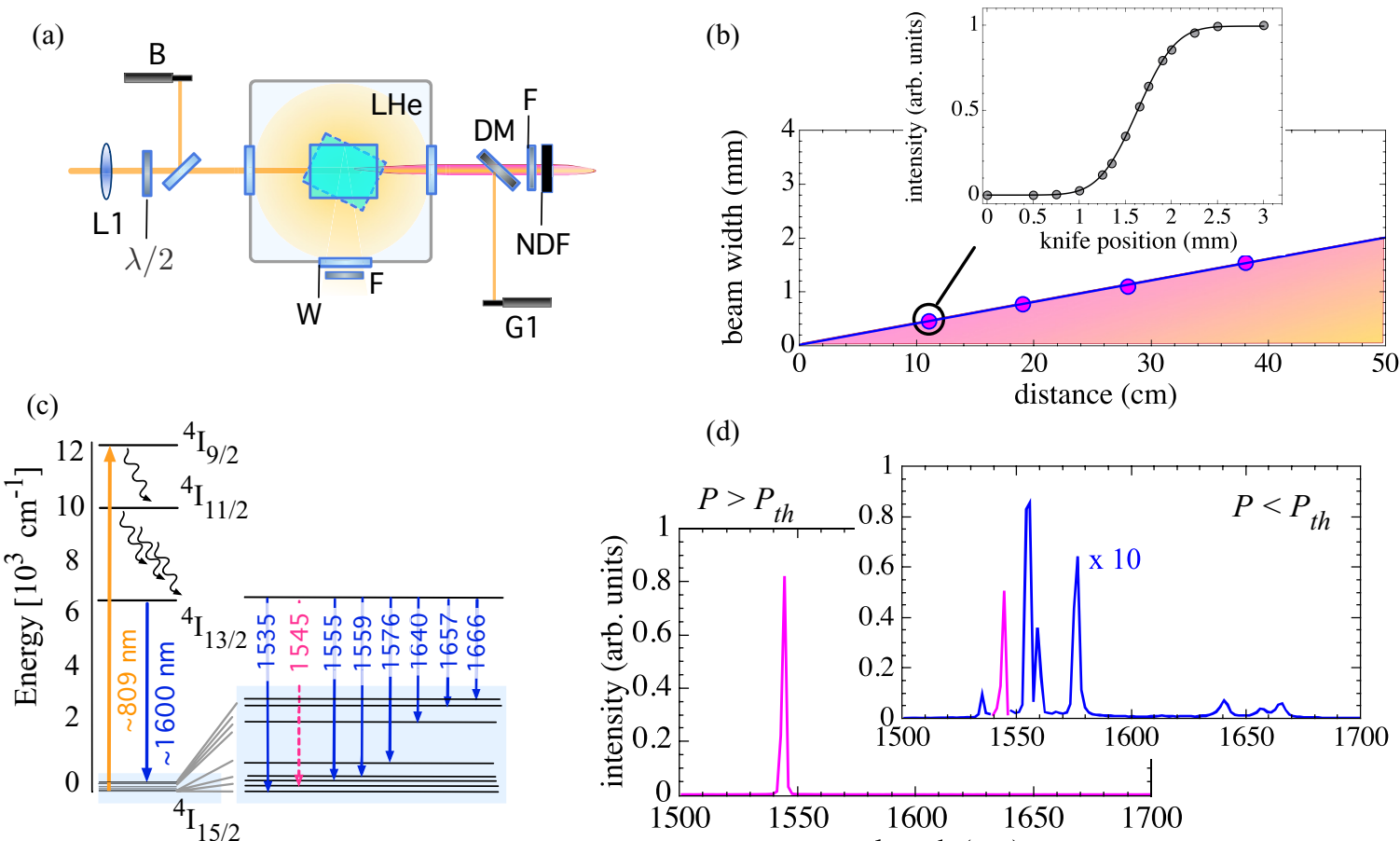

(d)

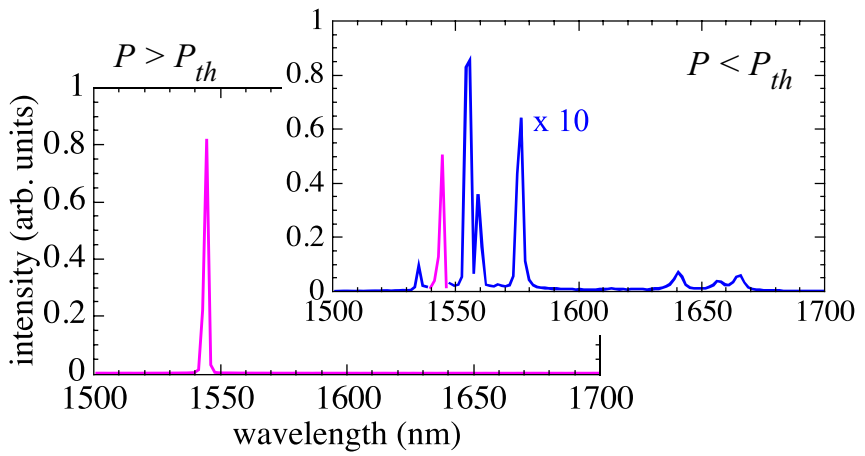

FIG. 1. (a) Experimental setup. A $0.1 \%$ concentration, $4 \times 5 \times 6.2 \mathrm{~mm}^{3}$-volume Er:YSO crystal is immersed in LHe and maintained at $1.6 \mathrm{~K}$. Er ions are indirectly pumped with a Ti:sapphire laser (stabilized ring cavity, $10 \mathrm{-MHz}$ linewidth) to the metastable level ${ }^{4} I_{13 / 2}$ shown in (c). The formation of the macroscopic dipole is accompanied by coherent emission in the forward direction. The incident laser beam is focused by lens L1 and its polarization can be rotated by means of half-wave plate $\lambda / 2$. A dichroic mirror (DM) reflects the pump laser light toward sensor G1 allowing for contextual laser absorption measurements. The forward light transmitted through DM is attenuated by a set of calibrated neutral density filters (NDF). Longpass filters (F) are used to remove stray laser light. Though optical window W we can monitor isotropic fluorescence or investigate off-axis SF emission. (b) Superfluorescent beam propagation and diffraction in the pencil-shaped sample geometry. The beam divergence angle $\theta_{d}$ is obtained by measuring with the knife-edge technique the forward beam radius at several distances from the crystal center, and the obtained $\theta_{d}$ value is compatible with diffraction from an aperture whose radius coincides with the measured laser beam waist $\omega_{0}=163 \mu \mathrm{m}$ at the crystal position $( \pm 1 \mathrm{~cm})$. [(c) and (d)] Emission spectral filtering. Above the threshold $P_{\mathrm{th}}$, superfluorescence emission takes place through the highest branching ratio transition, represented in the $\mathrm{Er}^{3+}$ energy levels scheme by the magenta dotted line arrow. The corresponding magenta line in the recorded $P>P_{\text {th }}$ spectrum is about $10^{5}$ times stronger than the overall emission related to the blue lines, whose intensity is instead comparable to that of the magenta line in the $P<P_{\text {th }}$ spectrum.

possibility to investigate this process would in fact pave the way to a new method of elusive particle detection, whereby a factor $N$ enhancement of rare events interaction rates might be accomplished in recently proposed upconversion schemes $[27,28]$.

\section{EXPERIMENTAL APPARATUS}

Atoms that can participate to the spontaneous formation of the macroscopic dipole are $\mathrm{Er}^{3+}$ ions, positioned at about 5-nm relative distance (corresponding to $0.1 \%$ atomic percentage substitution for $\mathrm{Y}^{3+}$ ) in a YSO crystalline host matrix cooled to 1.6 K. Site 1 [29] Er ions are incoherently excited by a continuous wave (cw) pump laser to the long-lived $\left(\tau_{0}=15.0 \pm 0.1 \mathrm{~ms}\right)^{4} I_{13 / 2}$ level via phonon-emitting steps as shown in Fig. 1(a).

The atomic sample is then automatically endowed with the shape of a long cylinder of length $L$, where $L=6.2 \mathrm{~mm}$ is the crystal length along the laser propagation direction, and transverse dimension $2 \omega_{0}$ ( $\omega_{0}$ laser beam waist), obeying the relation $L>\omega_{0} \gg \lambda$ (pencil-shaped sample), with $\lambda$ wavelength of the SF beam.

Clear evidence of the formation of a macroscopic dipole in our physical system is the observation of pulsed superradiant emission above a well-defined threshold value of inversion population density. When this condition is satisfied, we record a bright forward field whose emission spectrum displays spectral filtering, e.g., the natural multiline emission spectrum collapses to the highest branching ratio transition at $\lambda=$ $1.545 \mu \mathrm{m}$ [Fig. 1(d)].

Knife-edge measurements [Fig. 1(b)] show that the beam at $1.545 \mu \mathrm{m}$-wavelength emerging from the crystal is a Gaussian beam with far-field beam divergence $\theta_{d}=3.6 \pm$ $0.5 \mathrm{mrad}$. This value is comparable with the calculated diffraction angle $\theta_{D}=\lambda / 2 \omega_{0}=4.7 \mathrm{mrad}$ of the pencilshaped atomic sample, with $\omega_{0}=163 \mu \mathrm{m}$ measured pump beam waist. Such a remarkable control of the SF beam parameters is useful to distinguish emission supported by off-axial modes from diffraction of each mode propagating close to the cylinder axis [30-32]. The Fresnel factor $F=\pi \omega_{0}^{2} / L \lambda$ is the key parameter to set the conditions to enter such regimes 
giving a F-lobes pattern for $F>1$ and a single, wide-area lobe when $F<1$ [33]. In the present configuration $F \sim 9$, with emission intensity mainly concentrated within a single lobe as wide as $\theta_{D}$.

\section{OBSERVATION OF SF PULSES}

As is well known $[2,33]$, the pencil-shaped sample geometry not only determines the strong directionality of the SF light beam but also influences the temporal dynamics of the corresponding photon bunches. In fact, it mitigates the characteristic SF emission time $\tau_{R}$ through the geometrical factor $\mu=3 \Omega_{0} /(8 \pi)$, proportional to the diffraction solid angle $\Omega_{0}=\lambda^{2} /\left(\pi \omega_{0}^{2}\right)$ of the sample

$$
\tau_{R}=\bar{\tau} / \mu N,
$$

where we have introduced $\bar{\tau}=\tau_{0} / \beta$ as the inverse of the transition rate at $1545 \mathrm{~nm}$ wavelength [Fig. 1(c)]. The branching ratio $\beta=0.42$ is estimated by the spectra recorded below the SF threshold [Fig. 1(d)].

The SF full dynamics is enclosed in the hyperbolic secant shape of the emitted intensity [33],

$$
I(t)=\frac{h c}{\lambda} R_{p} \operatorname{sech}^{2}\left[\left(t-t_{0}\right) / 2 \tau_{R}\right],
$$

where $R_{p}$ is the peak photon output rate (i.e., pulse amplitude at $t=t_{0}$ ), proportional to $N^{2}$ as expected for SR.

Figure 2(a) unveils the stochastic nature of the phenomenon $[5,34,35]$ by showing three representative photon bunches recorded at fixed laser intensity. They have been selected from the several hundreds pulses analyzed to prepare the main plots. All the recorded pulses are well fitted by a pure sech-squared temporal profile, as confirmed by the linear regression in Fig. 2(c). From the pulses area we can infer $N$, provided the SF beam is properly coupled to small area, calibrated photodiodes and the optical losses are taken into account (as detailed in the Appendix). One cannot, however, directly associate one photon in the bunch with one atom participating to the macroscopic state, because the single pass gain $G$ of the SF pulse through the inverted medium must also be considered. We thus modify Eq. (2) to

$$
I(t)=\frac{h c}{\lambda} \frac{\mu \bar{N}\left(\bar{N}-N_{0}\right)}{4 \bar{\tau}} \operatorname{sech}^{2}\left[\left(t-t_{0}\right) / 2 \tau_{R}\right],
$$

having introduced the parameter $\bar{N}=N(1+G)=N+N_{0}=$ $4 \tau_{R} R_{p}$ representing the number of experimentally observed photons, which is greater than the number of photons $N$ emitted by the macroscopic dipole.

From Eqs. (2) and (3) we obtain:

$$
R_{p}=\frac{\mu \bar{N}\left(\bar{N}-N_{0}\right)}{4 \bar{\tau}},
$$

in which the superlinear scaling of the peak photon rate with $\bar{N}$ is explicit. By recalling that $\tau_{R}=\bar{\tau} /\left[\mu\left(\bar{N}-N_{0}\right)\right]$ and using Eq. (4), the superfluorescence time can be written as:

$$
\tau_{R}=\frac{N_{0}}{8 R_{p}}\left[1+\sqrt{1+16 R_{p} \frac{\bar{\tau}}{\mu N_{0}^{2}}}\right],
$$

Equations (4) and (5) connect the three observables $\bar{N}, \tau_{R}$, and $R_{p}$ and accurately fit the recorded data as shown in Fig. 2.
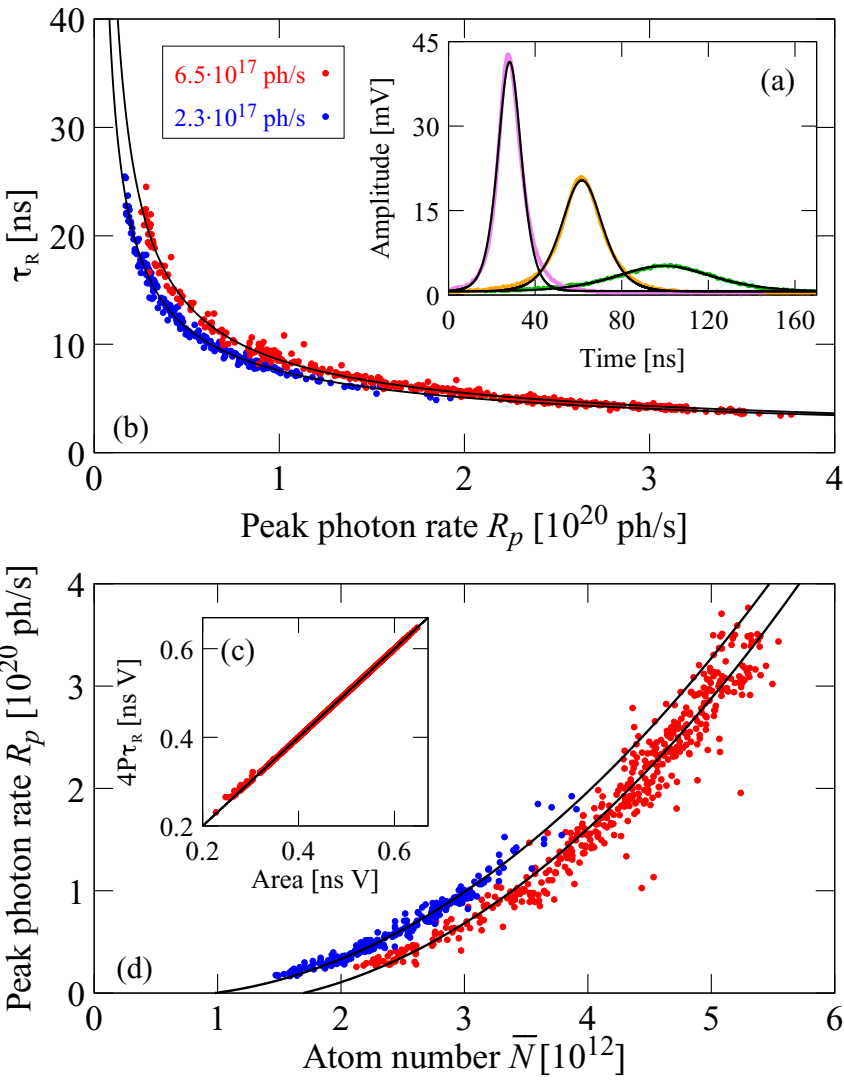

FIG. 2. Spontaneously generated superfluorescent pulses at different pump photon flux, corresponding to pump intensity values of 67 and $192 \mathrm{~W} / \mathrm{cm}^{2}$. (a) Representative individual time traces of photon bunches at different atom number $N \sim 5.4 \times 10^{12}$ (pink), $N \sim 4.6 \times 10^{12}$ (yellow), and $N \sim 2.6 \times 10^{12}$ (green). They have been recorded under identical excitation conditions and time shifted in the plot for representation purposes. The time dependence of the signal amplitude is very well fitted by a squared hyperbolic secant function. (b) Pulse duration $\tau_{R}$ versus peak photon rate $R_{p}$ for pumping rates. The black lines are fits in the form given by Eq. (3), giving an average $\mu=(2.4 \pm 0.1) \times 10^{-6}$, compatible with diffraction from an aperture of diameter $2 \omega_{0}=326 \mu \mathrm{m}$. (c) Nominal area of the fit function given by Eq. (2) versus integrated pulse area. Linear regression with unitary slope demonstrates pure SF dynamics for each recorded photon bunch. (d) Peak photon rate versus observed atom number. The recorded data scale superlinearly, as shown by the $N^{2}$ trend represented by the black line.

It is important to note that, due to the parametrization that we introduced to account for the medium gain, the recorded data are very well fitted by functional forms including two physical parameters, namely the excess photons $N_{0}$ and the geometrical factor $\mu$. Using an absolute calibration, we can estimate $\mu$ directly by the fitting procedure as $(2.4 \pm 0.1) \times$ $10^{-6}$, a value that favorably compares with that calculated by its definition $\mu=\frac{3}{8 \pi^{2}}\left(\frac{\lambda}{\omega_{0}}\right)^{2}=3.4 \times 10^{-6}$, given the onedimensional approximation [33]. Excess photon values $N_{0}$ of $(1.0 \pm 0.1) \times 10^{12}$ and $(1.7 \pm 0.1) \times 10^{12}$ are obtained for the two data series at different laser intensity, accordingly to the greater gain in the higher inversion medium. Note that data at higher intensity shown in Fig. 2(d) are characterized 

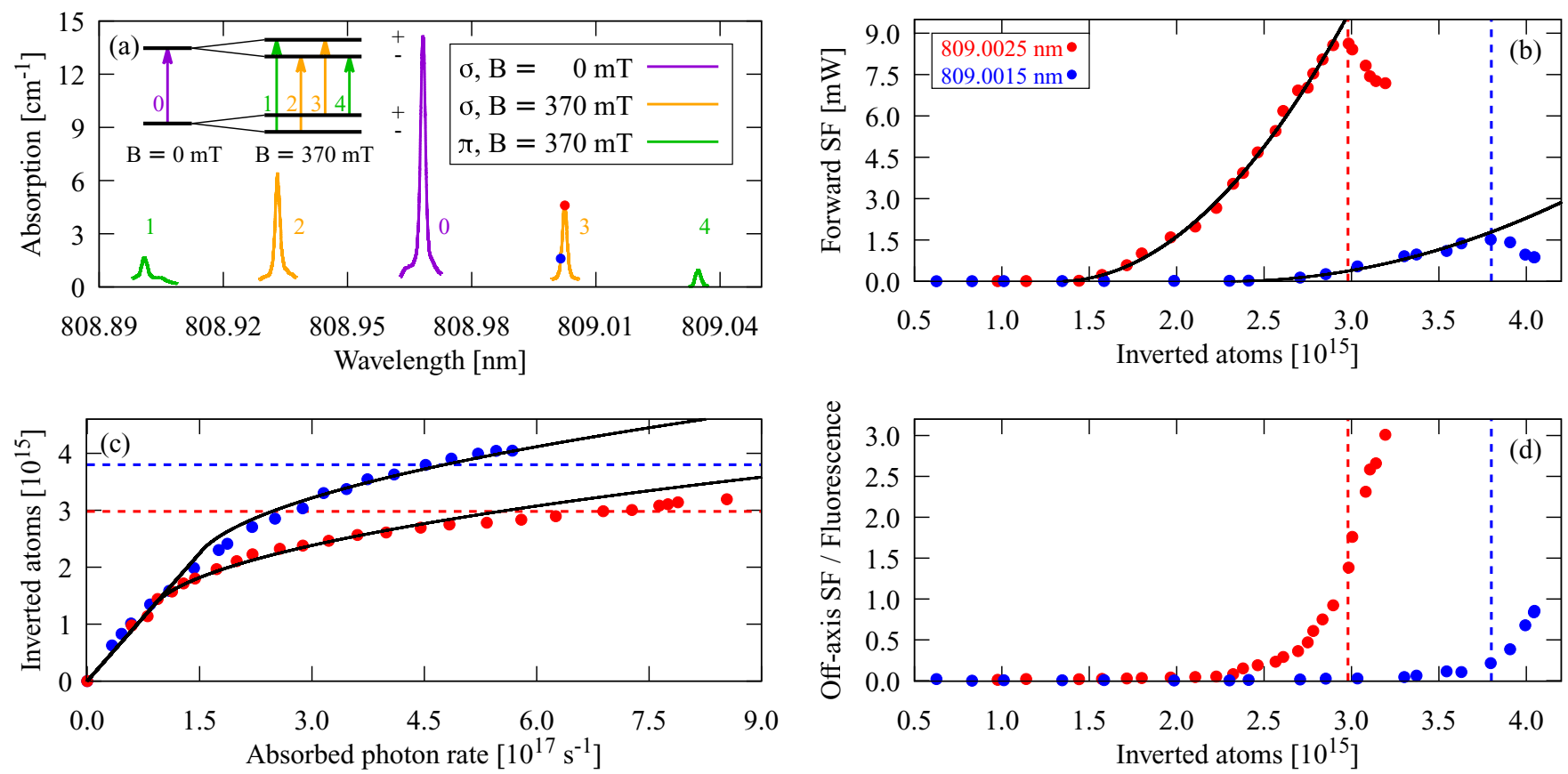

FIG. 3. (a) Absorption laser spectroscopy for the transition ${ }^{4} I_{15 / 2} \rightarrow{ }^{4} I_{9 / 2}$ for $\pi$ and $\sigma$ polarization with and without 370-mT magnetic field. (b) Forward (d) and off-axis average intensity of the superfluorescent emission for different longitudinal profiles of the inverted population density at absorption coefficients 1.6 and $4.6 \mathrm{~cm}^{-1}$, corresponding to centered (809.0025-nm) and slightly off-resonance (809.0015-nm) wavelengths, respectively [blue and red dots in Fig. 3(a)]. The dotted lines indicate when the off-axis emission is so important as to reverse the growth trend in the forward emission. The black lines are a parabolic fit to the data. (c) Data agreement with the steady-state rate equations analysis. Deviation from the initial linearity of the population inversion with pump absorption is due to the superfluorescence emission, which is more pronounced in the data recorded at resonance (red dot data).

by more pronounced deviations as one expects when intrinsic fluctuations in SF get amplified by a greater gain medium.

As in SF coherence is built up as the pulse is emitted, we can assume that the macroscopic dipole lifetime is given by the full width at half maximum $\left(\mathrm{FWHM}=3.52 \times \tau_{R}\right)$ of the observed pulses. Analysis of the forward emission shown in Fig. 2(d) definitely demonstrates macrocoherence involving more than $10^{12}$ atoms, and pulses durations up to FWHM $90 \mathrm{~ns}$ have been recorded.

\section{SF EMISSION AVERAGE INTENSITY}

As shown in Fig. 3(a), a sharp inhomogeneous absorption line of $600 \mathrm{MHz}$ is observed on the transition between the lowest Stark levels of the ${ }^{4} I_{15 / 2}$ and ${ }^{4} I_{9 / 2}$ manifolds. The absorption coefficient is $14.1 \mathrm{~cm}^{-1}$ at resonance, and we Zeeman split the levels with a 370-mT magnetic field to mitigate the pump laser absorption and accomplish a quasiuniform inverted population density. Forward emission is then investigated for two values of pump laser wavelength, centered and slightly off-resonance, as indicated by the blue and red dots in Fig. 3(a).

It is worth noting that the width of the transition at $1545 \mathrm{~nm}$ [indicated by the magenta arrow in Fig. 1(c)] is expected to be in the order of $1 \mathrm{GHz}$ [29], a value that does not comply with the recorded pulses duration (4-52 ns) according to $\tau_{R} \ll T_{2}^{*}$, unless collective emission by subsystems of atoms is considered. In fact, by monitoring the isotropic fluo- rescence and by taking into account the measured absorption [Fig. 3(a)], we estimate a steady-state inverted atom number $N_{1} \gtrsim 10^{15}$ in the ${ }^{4} I_{13 / 2}$ level, and we can thus state that a fraction $\sim 10^{-3}$ of the excited ions contribute to each macro dipole formation. We believe that this is an interesting aspect and that it is reasonable to explain the SF dynamics we observe in terms of self-selection of subensembles of atoms with closest transition wavelengths under cw excitation.

In Fig. 3(b) we report the plot we obtain when the forward SF beam average power is measured with a Ge power sensor for several laser pump intensities. The latter is expressed in inverted atom number using isotropic fluorescence spectra acquired at a spectrometer set in front of optical window $\mathrm{W}$ in Fig. 1(a). The integrated spectral intensity of the isotropic emission [seven blue lines in the full spectrum reported in Fig. 1(d)] is converted to inverted atoms number by imposing a linear dependence with slope $\tau_{0}$ to the data recorded at low pumping levels [to absorbed photon rate $10^{17} \mathrm{~s}^{-1}$ in Fig. 3(c)].

The main source of error (within few percentages) in the described conversion procedure is due to the losses introduced by the cryostat windows, estimated by comparison with the out-of-resonance absorption level. Note that the SF pulses rate is low $(\leqslant 100 \mathrm{kHz})$, and thus they do not significantly alter the excited level population density. We observe that the intensity of the forward emission is proportional to $\left(N_{1}-N_{10}\right)^{2}$ [Fig. 3(b)], where $N_{10}$ is the SF threshold atom number. Deviation from the predicted $N^{2}$ dependence at higher pumping levels is fully ascribed to off-axis, omnidirectional superfluorescence, as detailed in Sec. V. 


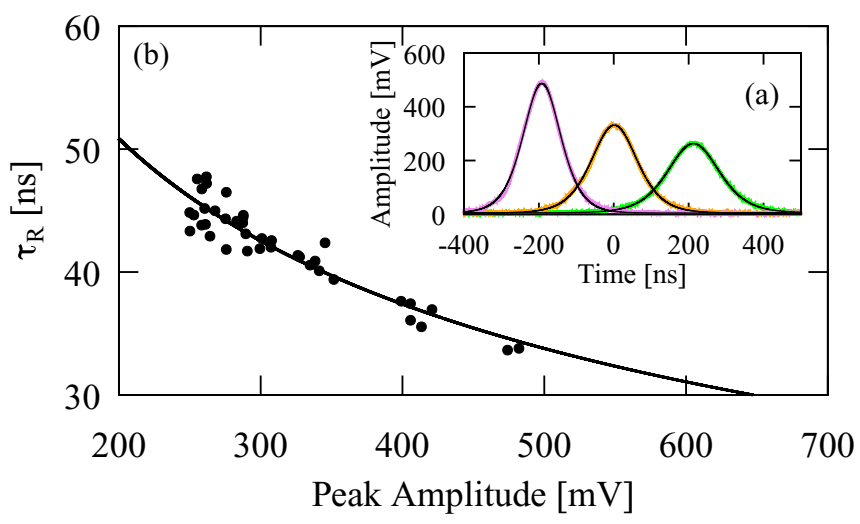

FIG. 4. Detection of transverse photon bunches with a fast photodiode (10-ns rise time, $0.8-\mathrm{mm}^{2}$ area) set in front of optical window at $\gtrsim 3 \mathrm{~cm}$ distance from $\mathrm{W}$ with no input coupling optics.

We indirectly analyze the efficiency of the cooperative emission process by reporting the inverted population vs. absorbed photons as shown in Fig. 3(c). As the ${ }^{4} I_{9 / 2}$ and ${ }^{4} I_{11 / 2}$ levels quickly relax via efficient multiphonon relaxation to the ${ }^{4} I_{13 / 2}$, its level population number $N_{1}$ can be thoroughly described by a single, steady-state rate equation:

$$
\begin{gathered}
\Phi_{\mathrm{abs}}-N_{1} / \tau_{0}=0 \quad\left(N_{1}<N_{10}\right), \\
\Phi_{\mathrm{abs}}-N_{1} / \tau_{0}-b\left(N_{1}-N_{10}\right)^{2}=0 \quad\left(N_{1}>N_{10}\right),
\end{gathered}
$$

in which the $b$ coefficient quantifies the level depletion by the cooperative process, $N_{10}$ is the SF threshold inferred from the plot in Fig. 3(b) and $\Phi_{\mathrm{abs}}$ is the absorbed photon rate. The solution of Eq. (6) predicts a linear dependence of the inverted population vs. absorbed photons when $N_{1}$ is below the SF threshold $N_{10}$ and a marked $\sqrt{\Phi_{\text {abs }}}$ trend otherwise. As long as the off-axis emission is not relevant, data reported in Fig. 3(c) are well fitted by this solution, and the gap to the projected linear fluorescence value gives the fraction of ions that is diverted to SF, as high as $74 \%$ over the photon bunch duration timescale.

\section{OFF-AXIS SF EMISSION}

For increasing values of inverted population, the same phenomenon of spectral filtering, reported previously for the emitted radiation in the forward direction, is observed with the spectrometer input set in front of window W [Fig. 1(a)], indicating transverse SF photon bunching.

Inset of Fig. 4 shows three time-shifted representative photon bunches. The recorded transverse pulses are well fitted by the same pure sech-squared temporal profile used for the forward bunches. The temporal dynamics of these off-axis photon bunches satisfies Eqs. (3) and (5), even though with a larger characteristic $\tau_{R}$ compared to the forward pulses due to the much smaller number of available atoms along the cylindric sample transverse dimension $2 \omega_{0}<L$. In Fig. 3(d) we report the ratio of these coherent pulses average intensity (a single line in the spectrum) with the incoherent fluorescence emission $(7+1$ lines in the spectrum), having subtracted from the first the component due to the scattered SF forward intensity. Deviation from the predicted $N^{2}$ dependence at the highest pumping levels in Fig. 3(b) is fully ascribed to offaxis, omnidirectional superfluorescence, as confirmed by the plot in Fig. 3(d). An increasing fraction of the atomic sample is involved in this transverse cooperative emission to such an extent that the forward emission trend gets inverted in both the data series obtained for two different population inversion profiles [see Fig. 3(b)]. This is a new aspect that is of importance for the present work aims, previously not reported in experiments performed in the pencil-shaped sample geometry $[5,36,37]$.

\section{CONCLUSIONS AND OUTLOOK}

We have reported unambiguous evidence of superfluorescence achieved by incoherently seeding a cw population inversion on the 1.54- $\mu \mathrm{m}$ transition in Er:YSO. The observed cleanest sech-squared light pulses differ from the complex superradiance dynamics observed by several groups, which are affected by ringing effects, asymmetry with long tails and multiple structures, thus showing that coherent ringing is not an intrinsic feature of SF in solid-state extended media, as has generally come to be believed [30,38].

The peak photon rate $R_{p}$ is demonstrated to scale quadratically with atom number and the light pulses width follows the predicted $1 / \sqrt{R_{p}}$ trend, as long as the high gain of the inverted medium is taken into account.

Superradiant pulses being emitted at a rate a million times faster than the incoherent spontaneous emission time have been reported, demonstrating self-driven atom dynamics with no cavity mediation nor interaction engineering to further complicate the description and limit the number of involved dipoles. The key $N$-squared scaling of the emission, which distinguishes SF from amplified spontaneous emission [23], is also found when we integrate the energy of thousands forward photon bunches. Interestingly, we have registered SF pulses that propagate orthogonally to the cylinder-axis atomic sample, indicating adequate optical thickness for SF also in the transverse direction for our highest pumping levels $\left(\sim 0.3 \mathrm{~kW} / \mathrm{cm}^{2}\right)$. Thus far, omnidirectional emission had only been reported in a $\mathrm{Rb}$ vapor sample, even though as a result of four-wave mixing [39].

A quantitative approach to SF has been developed, based on a single, simple rate equation at the steady state for the fluorescent level ${ }^{4} I_{13 / 2}$, which includes a superfluorescencerelated term weighting both the pulse rate and amplitude. Still, in the framework of ultranarrow laser development, it has been emphasized how pulsed emission is not an intrinsic property of superradiance and that operation in a continuous manner, with pump lasers applied to return the atoms to the excited state, is a viable solution to reach the $\mathrm{mHz}$ linewidth laser [6-8]. The results of our SF beam propagation study finally comply with the diffraction-based theoretical description given long ago by Gross and Haroche [33] about superfluorescence by an extended sample of atoms. This study is of importance for the design of a resonant repumping cavity.

From the properties of the emitted superradiant field, we infer the spontaneous formation of a macroscopically 


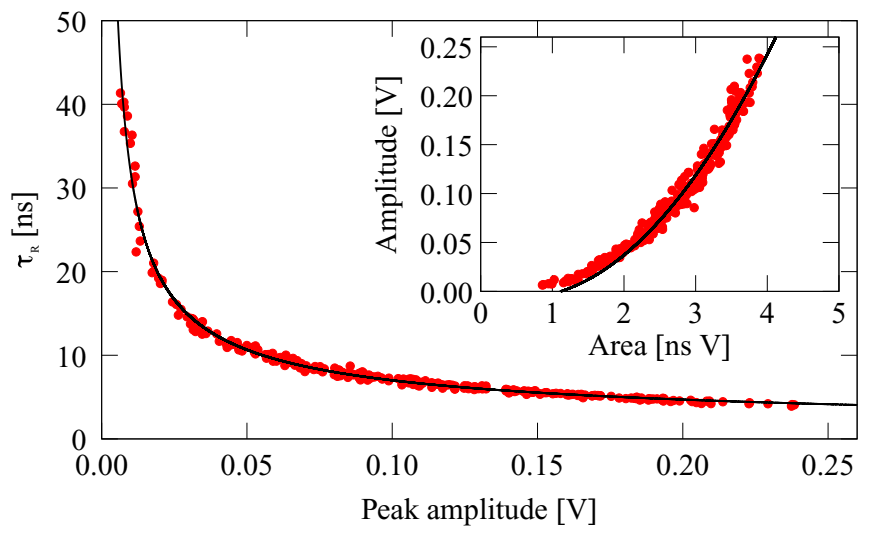

FIG. 5. Temporal dynamics recorded at an ultrafast (25-ps rise time) InGaAs photodiode.

extended coherent state, whose key parameters, namely the number of involved atoms $\left(\sim 4 \times 10^{12}\right)$ and lifetime $(\sim 100 \mathrm{~ns})$, promise applicability as a test bed for quantum optics effects. With the numbers already at hand in this work, a $\sim 1 \%$ duty cycle coherent sensor is envisaged, capable at coherently amplifying the smallest of signals, with an overall intrinsic gain of $4 \times 10^{12}$ [40].

\section{ACKNOWLEDGMENT}

We thank L. A. Lugiato for helpful discussions and critical reading of the manuscript.

\section{APPENDIX}

To operate the photodiodes in their linear response range the input forward SF light beam is attenuated by three neutral density filters, for a total transmittance of $2.3 \times 10^{-5}$. The SF beam is first collimated and then focused to a beam diameter compatible with full photon bunch detection at the fast photodiode area (10-ns rise time, $0.8-\mathrm{mm}^{2}$ area). The number of photons reported in the horizontal axis of Fig. 2(d) is thus obtained by computing the pulse area and taking into account the PD responsivity. Acquisition at a 6-GHz digital sampling oscilloscope and analysis of the same pulses at ultrafast photodiode (25-ps rise time, $10^{-3}-\mathrm{mm}^{2}$ area) ensured no distortion of the temporal dynamics recorded at the fast photodiodes as proven in Fig. 5. In addition, this result shows that the same dynamics reported in Fig. 2 is observed also when we sample a fraction of the SF beam.
[1] R. H. Dicke, Coherence in spontaneous radiation processes, Phys. Rev. 93, 99 (1954).

[2] N. E. Rehler and J. H. Eberly, Superradiance, Phys. Rev. A 3, 1735 (1971).

[3] R. Bonifacio and L. A. Lugiato, Cooperative radiation processes in two-level systems: Superfluorescence, Phys. Rev. A 11, 1507 (1975).

[4] N. Skribanowitz, I. P. Herman, J. C. MacGillivray, and M. S. Feld, Observation of Dicke Superradiance in Optically Pumped HF Gas, Phys. Rev. Lett. 30, 309 (1973).

[5] R. Florian, L. O. Schwan, and D. Schmid, Time-resolving experiments on dicke superfluorescence of $\mathrm{O}_{2}^{-}$centers in $\mathrm{KCl}$. Two-color superfluorescence, Phys. Rev. A 29, 2709 (1984).

[6] D. Meiser, J. Ye, D. R. Carlson, and M. J. Holland, Prospects for a Millihertz-Linewidth Laser, Phys. Rev. Lett. 102, 163601 (2009).

[7] D. Meiser and M. J. Holland, Steady-state superradiance with alkaline-earth-metal atoms, Phys. Rev. A 81, 033847 (2010).

[8] J. G. Bohnet, Z. Chen, J. M. Weiner, D. Meiser, M. J. Holland, and J. K. Thompson, A steady-state superradiant laser with less than one intracavity photon, Nature 484, 78 (2012).

[9] M. A. Norcia, M. N. Winchester, J. R. K. Cline, and J. K. Thompson, Superradiance on the millihertz linewidth strontium clock transition, Sci. Adv. 2, e1601231 (2016).

[10] M. A. Norcia, J. R. K. Cline, J. A. Muniz, J. M. Robinson, R. B. Hutson, A. Goban, G. E. Marti, J. Ye, and J. K. Thompson, Frequency Measurements of Superradiance from the Strontium Clock Transition, Phys. Rev. X 8, 021036 (2018).

[11] G. Rainò, M. A. Becker, M. I. Bodnarchuk, R. F. Mahrt, M. V. Kovalenko, and T. Stöferle, Superfluorescence from lead halide perovskite quantum dot superlattices, Nature 563, 671 (2018).
[12] A. Angerer, K. Streltsov, T. Astner, S. Putz, H. Sumiya, S. Onoda, J. Isoya, W. J. Munro, K. Nemoto, J. Schmiedmayer, and J. Majer, Superradiant emission from color centres in diamond, Nat. Phys. 14, 1168 (2018).

[13] K. Cong, Q. Zhang, Y. Wang, G. T. Noe, A. Belyanin, and J. Kono, Dicke superradiance in solids [invited], J. Opt. Soc. Am. B 33, C80 (2016).

[14] T. Laurent, Y. Todorov, A. Vasanelli, A. Delteil, C. Sirtori, I. Sagnes, and G. Beaudoin, Superradiant Emission from a Collective Excitation in a Semiconductor, Phys. Rev. Lett. 115, 187402 (2015).

[15] G. Timothy Noe II, J.-H. Kim, J. Lee, Y. Wang, A. K. Wójcik, S. A. McGill, D. H. Reitze, A. A. Belyanin, and J. Kono, Giant superfluorescent bursts from a semiconductor magneto-plasma, Nat. Phys. 8, 219 (2012).

[16] Y. D. Jho, X. Wang, J. Kono, D. H. Reitze, X. Wei, A. A Belyanin, V. V. Kocharovsky, Vl. V. Kocharovsky, and G. S. Solomon, Cooperative Recombination of a Quantized HighDensity Electron-Hole Plasma in Semiconductor Quantum Wells, Phys. Rev. Lett. 96, 237401 (2006).

[17] C. W. Thiel, T. Böttger, and R. L. Cone, Rare-earth-doped materials for applications in quantum information storage and signal processing, J. Lumin. 131, 353 (2011).

[18] R. M. Macfarlane, T. L. Harris, Y. Sun, R. L. Cone, and R. W. Equall, Measurement of photon echoes in $\mathrm{Er}: \mathrm{Y}_{2} \mathrm{SiO}_{5}$ at $1.5 \mu \mathrm{m}$ with a diode laser and an amplifier, Opt. Lett. 22, 871 (1997).

[19] M. Rančić, M. P. Hedges, R. L. Ahlefeldt, and M. J. Sellars, Coherence time of over a second in a telecom-compatible quantum memory storage material, Nat. Phys. 14, 50 (2017).

[20] X. Fernandez-Gonzalvo, Y.-H. Chen, C. Yin, S. Rogge, and J. J. Longdell, Coherent frequency up-conversion of microwaves to 
the optical telecommunications band in an Er:YSO crystal, Phys. Rev. A 92, 062313 (2015).

[21] S. Probst, A. Tkalčec, H. Rotzinger, D. Rieger, J.-M. Le Floch, M. Goryachev, M. E. Tobar, A. V. Ustinov, and P. A. Bushev, Three-dimensional cavity quantum electrodynamics with a rare-earth spin ensemble, Phys. Rev. B 90, 100404(R) (2014).

[22] S. Probst, H. Rotzinger, A. V. Ustinov, and P. A. Bushev, Microwave multimode memory with an erbium spin ensemble, Phys. Rev. B 92, 014421 (2015).

[23] M. S. Malcuit, J. J. Maki, D. J. Simkin, and R. W. Boyd, Transition from Superfluorescence to Amplified Spontaneous Emission, Phys. Rev. Lett. 59, 1189 (1987).

[24] A. Ishikawa, K. Miyajima, M. Ashida, T. Itoh, and H. Ishihara, Theory of superfluorescence in highly inhomogeneous quantum systems, J. Phys. Soc. Jpn. 85, 034703 (2016).

[25] K. D. B. Higgins, S. C. Benjamin, T. M. Stace, G. J. Milburn, B. W. Lovett, and E. M. Gauger, Superabsorption of light via quantum engineering, Nat. Commun. 5, 4705 (2014).

[26] W. M. Brown and E. M. Gauger, Light-harvesting with guideslide superabsorbing condensed-matter nanostructures, J. Phys. Chem. Lett. 10, 4323 (2019).

[27] C. Braggio, G. Carugno, F. Chiossi, A. D. Lieto, M. Guarise, P. Maddaloni, A. Ortolan, G. Ruoso, L. Santamaria, J. Tasseva, and M. Tonelli, Axion dark matter detection by laser induced fluorescence in rare-earth doped materials, Sci. Rep. 7, 15168 (2017).

[28] P. Sikivie, Axion Dark Matter Detection using Atomic Transitions, Phys. Rev. Lett. 113, 201301 (2014).

[29] T. Böttger, C. W. Thiel, Y. Sun, and R. L. Cone, spectroscopy and dynamics of $\mathrm{Er}^{3+}: \mathrm{Y}_{2} \mathrm{Sio}_{5}$ at $1.5 \mu \mathrm{m}$, Phys. Rev. B 74, 075107 (2006).
[30] D. J. Heinzen, J. E. Thomas, and M. S. Feld, Coherent Ringing in Superfluorescence, Phys. Rev. Lett. 54, 677 (1985).

[31] F. P. Mattar, H. M. Gibbs, S. L. McCall, and M. S. Feld, Transverse Effects in Superfluorescence, Phys. Rev. Lett. 46, 1123 (1981).

[32] M. G. Benedict, A. M. Ermolaev, V. A. Malyshev, I. V. Sokolov, and E. D. Trifonov, Super-radiance Multiatomic Coherent Emission (Taylor \& Francis Group, London, 1996).

[33] M. Gross and S. Haroche, Superradiance: An essay on the theory of collective spontaneous emission, Phys. Rep. 93, 301 (1982).

[34] Q. H. F. Vrehen, M. F. H. Schuurmans, and D. Polder, Superfluorescence: Macroscopic quantum fluctuations in the time domain, Nature 285, 70 (1980).

[35] Q. H. F. Vrehen and J. J. der Weduwe, Quantum fluctuations in superfluorescence delay times, Phys. Rev. A 24, 2857 (1981).

[36] M. Gross, C. Fabre, P. Pillet, and S. Haroche, Observation of Near-Infrared Dicke Superradiance on Cascading Transitions in Atomic Sodium, Phys. Rev. Lett. 36, 1035 (1976).

[37] H. M. Gibbs, Q. H. F. Vrehen, and H. M. J. Hikspoors, SinglePulse Superfluorescence in Cesium, Phys. Rev. Lett. 39, 547 (1977).

[38] C. Greiner, B. Boggs, and T. W. Mossberg, Superradiant Emission Dynamics of an Optically thin Material Sample in a Short-Decay-Time Optical Cavity, Phys. Rev. Lett. 85, 3793 (2000).

[39] A. I. Lvovsky, S. R. Hartmann, and F. Moshary, Omnidirectional Superfluorescence, Phys. Rev. Lett. 82, 4420 (1999).

[40] N. W. Carlson, D. J. Jackson, A. L. Schawlow, M. Gross, and S. Haroche, Superradiance triggering spectroscopy, Opt. Commun. 32, 350 (1980). 